\title{
Perceptions de finissants en ÉPS au regard de l'insertion professionnelle
}

Johanne Grenier, Marie-Claude Rivard, Charlotte Beaudoin, Sylvain Turcotte et Mylène Leroux

\section{(2) OpenEdition}

Édition électronique

URL : http://journals.openedition.org/ripes/713

DOI : 10.4000/ripes.713

ISSN : 2076-8427

Éditeur

Association internationale de pédagogie universitaire

Référence électronique

Johanne Grenier, Marie-Claude Rivard, Charlotte Beaudoin, Sylvain Turcotte et Mylène Leroux, "Perceptions de finissants en ÉPS au regard de l'insertion professionnelle », Revue internationale de pédagogie de l'enseignement supérieur [En ligne], 29(1) | 2013, mis en ligne le 15 juin 2013, consulté le 08 septembre 2020. URL : http://journals.openedition.org/ripes/713 ; DOI : https://doi.org/10.4000/ ripes.713

Ce document a été généré automatiquement le 8 septembre 2020

Article L.111-1 du Code de la propriété intellectuelle. 


\title{
Perceptions de finissants en ÉPS au regard de l'insertion professionnelle
}

\author{
Johanne Grenier, Marie-Claude Rivard, Charlotte Beaudoin, Sylvain \\ Turcotte et Mylène Leroux
}

\section{Introduction}

1 L'insertion professionnelle est une étape cruciale et souvent ardue de la carrière des enseignants (Tait, 2008). Il semble que cette étape soit plus complexe qu'auparavant considérant les conditions de précarité qui la décrivent (Portelance, Mukamurera, Martineau, \& Gervais, 2008). En outre, même si les débutants vivent une période intense d'apprentissage et d'adaptation (Feiman-Nemser, Schwille, Carver, \& Yusko, 1999), on attend d'eux qu'ils assument les mêmes responsabilités que les enseignants d'expérience (Curtner-Smith, 2001; Fantilli \& McDougall, 2009; Le Maistre \& Paré, 2010). Qui plus est, ils se retrouvent souvent avec les tâches les plus lourdes et les plus complexes (COFPE, 2002; CSE, 2004). De plus, le passage du statut de stagiaire à celui d'enseignant confronte les débutants à la complexité du métier et à certaines réalités qu'ils n'ont pas nécessairement vécues en stage (Mukamurera, 2005). La complexité de la tâche renvoie à la maîtrise de nouvelles responsabilités (Lamarre, 2010) et le manque d'expérience et d'aisance dans la profession se fait alors sentir (Mukamurera, Bourque, \& Ntebutse, 2010). Ces conditions suscitent beaucoup de changements pour les débutants et font en sorte qu'ils sont ainsi souvent placés en situation de survie. Cela a un impact négatif sur leur efficacité, d'où le sentiment d'incompétence ressenti par certains d'entre eux (Mukamurera, 2005). Une étude de Hoy et Spero (2005) révèle d'ailleurs que même si le sentiment d'efficacité de futurs enseignants augmente significativement au cours de la formation initiale, il diminue de manière significative au cours de la première année d'insertion dans la profession.

2 Bien que l'on puisse constater que plusieurs études ont identifié les défis de l'insertion professionnelle en enseignement, peu d'entre elles se sont intéressées spécifiquement aux enseignants en éducation physique et à la santé (ÉPS) (Blankenship \& Coleman, 
2009). Spallanzani et ses collaborateurs (2012) soutiennent pourtant qu'il existe des différences notables dans les expériences d'insertion professionnelle vécues par ces enseignants de même que Stroot et Ko (2006) soulignent l'importance d'étudier le "scénario unique» de l'insertion professionnelle des enseignants en éducation physique.

\section{Contexte}

3 L'insertion professionnelle fait l'objet de nombreux travaux, mais constitue un intérêt de recherche grandissant depuis le virage de la réforme québécoise au début des années 2000. Le Ministère de l'Éducation du Québec (MEQ) a entrepris en 2001 une importante réforme qui se distingue des précédentes par une approche par compétences. Depuis, nous assistons à d'importants changements dans le système de l'éducation qui touchent tous les ordres d'enseignement, notamment la formation initiale des enseignants. De fait, le cursus universitaire québécois en enseignement est depuis associé à un référentiel de douze compétences professionnelles (MEQ, 2001) qui a obligé les institutions de formation à assurer le profil de sortie tel qu'attendu de tout nouvel enseignant. Le référentiel est élaboré autour de quatre catégories: 1) fondements, 2) acte d'enseigner, 3) contexte scolaire et social et 4) identité professionnelle traduisant ainsi un champ de compétences élargi et requis chez tous les nouveaux enseignants. L'éducation physique et à la santé comme programme de formation universitaire n'a pas échappé à cette vague de changements. La formation initiale ainsi revisitée a inévitablement engendré une mouvance dans le processus de l'insertion professionnelle. Quel est donc l'état des lieux de l'insertion professionnelle en ÉPS au tournant d'une décennie de grands changements éducatifs ? Voilà la grande question posée dans le cadre de cet article qui sera abordée à travers le filtre du référentiel des compétences professionnelles (MEQ, 2001), tout en cherchant à alimenter la réflexion entourant la formation universitaire offerte aux futurs enseignants.

\section{L'insertion professionnelle en ÉPS}

Quelques études décrivent les particularités des premières années d'expérience des enseignants en éducation physique. À l'instar de Vonk (1995), les résultats de ces études abordent l'insertion professionnelle selon trois niveaux. D'abord, le niveau personnel comprend l'information directement reliée à l'enseignant en tant que personne, à l'image qu'il a de lui-même ainsi qu'au sentiment qu'il éprouve à l'égard de son insertion professionnelle. Puis, le niveau professionnel regroupe les connaissances et les pratiques enseignantes que Vonk (1995) divise en trois parties : a) la connaissance des contenus enseignés, b) les habiletés de gestion des élèves et c) les habiletés d'enseignement. Enfin, le troisième niveau correspond à trois environnements soit a) l'école, b) le système éducatif et c) le système social que Vonk (1995) représente comme des cercles concentriques autour de l'enseignant. 


\subsection{Le niveau personnel}

5 Lorsque les nouveaux enseignants en ÉPS s'expriment sur les difficultés rencontrées lors de leur première année d'enseignement, ils soulignent une grande fatigue (Hill \& Brodin, 2004 ; Shoval, Erlich, \& Fejgin, 2010) surtout due aux exigences physiques de leur tâche (Hand, 2007; Mohr \& Townsend, 2001) et à l'inquiétude qui plane constamment à propos de leur précarité d'emploi (Hand, 2007). Les enseignants débutants en ÉPS rapportent qu'ils doivent laisser croire à leur entourage que tout va bien (Shoval, Erlich, \& Fejgin, 2010), ce qui engendre aussi une forme d'isolement (Curtner-Smith, 2001; Mohr \& Townsend, 2001) puisqu'ils hésitent à demander de l'aide à leurs collègues lorsque des difficultés surviennent. Cet isolement plus ou moins volontaire peut s'amplifier par le fait que les nouveaux enseignants en ÉPS sont, dans la plupart des cas, installés à proximité des infrastructures sportives, ce qui les éloigne des autres enseignants (Eldar, Nable, Schechter, \& Marzin, 2003). Cependant, Shoval, Erlich et Fejgin (2009) décrivent les nouveaux enseignants en ÉPS comme des personnes ambitieuses, vigoureuses, dédiées et flexibles, qui n'hésitent pas à s'investir dans leur nouvelle profession, à travailler beaucoup et à innover, tout en respectant la culture de l'école afin de s'insérer subtilement à l'équipe d'enseignants.

\subsection{Le niveau professionnel}

Les difficultés rencontrées par les nouveaux enseignants en ÉPS sont majoritairement de niveau professionnel (Shoval, Erlich, \& Fejgin, 2010). Une première difficulté exprimée réside dans le choix des contenus à enseigner (Hill \& Brodin, 2004), la transposition didactique de ces contenus (Roux-Perez, 2003) ainsi que leur adaptation pour les élèves présentant des besoins particuliers (Fejgin, Talmor, \& Erlich, 2005 ; Hill \& Brodin, 2004). Une très grande part de leurs difficultés a trait à la gestion des élèves dont la gestion des comportements perturbateurs (Roux-Perez, 2003 ; Shoval, Erlich, \& Fejgin, 2009) et à la motivation des élèves (Hill \& Brodin, 2004). D’ailleurs, les nouveaux enseignants en ÉPS rapportent une difficulté spécifique à l'enseignant de l'ÉPS en soulignant l'importance de gérer le groupe d'élèves dans un environnement à risque dans lequel ils doivent assurer leur sécurité (Hand, 2007). Enfin, lors de leur première année, les enseignants en ÉPS perçoivent un décalage important entre la théorie et la pratique (Mohr \& Townsend, 2001; Roux-Perez, 2003) au point de percevoir une régression de leurs compétences (Shoval, Erlich, \& Fejgin, 2010). Ils se sentent dépourvus par rapport aux parents de leurs élèves (Hill \& Brodin, 2004), à l'évaluation des élèves (Hill \& Brodin, 2004) et s'aperçoivent soudainement que leur tâche est plutôt répétitive (Macdonald, 1995). Néanmoins, certaines études soulèvent que la formation initiale est une variable importante d'une insertion professionnelle réussie (CurtnerSmith, 1998). En particulier, lorsqu'elle leur permet d'acquérir une maîtrise des contenus à enseigner, une capacité à innover et qu'elle favorise le développement des valeurs associées à l'ÉPS que les enseignants pourront transmettre aux élèves (Shoval, Erlich, \& Fejgin, 2010).

\subsection{Le niveau environnemental}

7 En reprenant le premier cercle proposé par Vonk (1995) soit l'école, les études rapportent que les enseignants en ÉPS perçoivent que les relations avec certains 
collègues enseignants de même discipline sont difficiles. Parfois, ils doivent paraître, penser et agir selon une culture de l'école qui ne leur convient pas, principalement lorsque leurs collègues ont conservé des approches pédagogiques et des contenus vieillots (Sirna, Tinning, \& Rossi, 2008). Pour leur part, Shoval, Erlich, \& Fejgin (2009) rapportent que les enseignants en ÉPS considèrent difficile de respecter les procédures habituelles de l'école qu'ils apprennent à leurs dépens puisqu'ils ignorent souvent ce qui se passe en dehors de leurs lieux d'enseignement et qu'ils perçoivent généralement comme étant très ambiguës. La perception d'une surveillance constante de la part de leurs supérieurs (Macdonald, 1995) associée à un manque de soutien de l'administration (Hill \& Brodin, 2004) et au constat d'un faible pouvoir de décision (Macdonald, 1995 ; Mohr \& Townsend, 2001) s'ajoutent aux difficultés évoquées par les nouveaux enseignants en ÉPS. Associées à ce cercle de l'école, des difficultés supplémentaires ont été recensées par d'autres chercheurs par rapport à l'environnement immédiat des enseignants en ÉPS, en l'occurrence les différents plateaux d'enseignement dans lesquels ils exercent. En effet, plusieurs difficultés rencontrées se rapportent au manque de matériel sportif ou didactique de qualité ainsi qu'à des installations sportives inadéquates (Blankenship \& Coleman, 2009). Il semble aussi que le temps dévolu à l'ÉPS soit aussi considéré comme une difficulté chez les nouveaux enseignants en ÉPS; ils soulignent les multiples modifications à l'horaire de leurs cours occasionnées par l'utilisation des plateaux sportifs pour le dîner des élèves ou les événements spéciaux (Hand, 2007).

8 Le système éducatif, qui constitue le deuxième cercle de Vonk (1995), semble peu préoccupant pour les nouveaux enseignants en ÉPS si ce n'est qu'ils connaissent très peu les impacts du système éducatif sur leur travail.

9 Par contre, la difficulté majeure issue du système social, présenté comme le troisième cercle de Vonk (1995), concerne le peu de valeur accordée par la société à l'enseignement de l'ÉPS. Présent dès les premières études sur les difficultés rencontrées par les nouveaux enseignants en ÉPS (Smyth, 1995 ; Solmon, Worthy, \& Carter, 1993 ; Stroot, 2003), ce problème de légitimité accordée à l'ÉPS est toujours perçu comme une difficulté importante (Blankenship \& Coleman, 2009 ; Hand, 2007 ; Hill \& Brodin, 2004 ; Mohr \& Townsend, 2001 ; Shoval, Erlich, \& Fejgin, 2009; Sirna, Tinning, \&Rossi, 2008 ; Stroot \& Ko, 2006). Plusieurs nouveaux enseignants en ÉPS sont surpris de constater le peu de valeur accordée à l'ÉPS autant par leurs collègues, les parents que par les élèves (Wright, 2001).

10 Telles que le décrivent les précédentes études, les premières années d'insertion professionnelle des enseignants en ÉPS sont teintées de difficultés importantes qu'ils doivent surmonter pour assurer leur survie professionnelle. Pourtant, beaucoup d'enseignants en ÉPS réussissent leur insertion professionnelle dans des milieux similaires à ceux que d'autres ont choisi de quitter. Hebert et Worthy (2001) émettent l'hypothèse que les expériences antérieures, la formation et les caractéristiques du milieu d'accueil influencent non seulement le succès de l'enseignant, mais aussi l'interprétation qu'il fait du contexte et de ses propres performances d'enseignement.

11 Cette littérature documente l'insertion professionnelle d'enseignants débutants en ÉPS et met en exergue un grand nombre de variables susceptibles d'influencer ce passage crucial situé à la croisée de la formation initiale et du début de carrière. C'est dans ce contexte que la présente étude cherche à documenter le phénomène de l'insertion professionnelle au Québec en s'intéressant aux perceptions des nouveaux enseignants 
en ÉPS en regard des compétences professionnelles qu'ils estiment les plus déterminantes dans l'exercice de leur profession ainsi que du niveau de maîtrise de chacune d'entre-elles.

\section{Cadre conceptuel}

Rappelons que la formation initiale des enseignants au Québec, quelle que soit la discipline enseignée, s'appuie sur le référentiel des douze compétences professionnelles en enseignement (MEQ, 2001). Les futurs enseignants en ÉPS ont vécu, au cours de leur formation, différentes activités académiques leur permettant d'atteindre le niveau de maîtrise tel qu'attendu selon les compétences du référentiel. Ce sont ces mêmes compétences qui encadrent l'évaluation des finissants et permettent d'accorder un permis d'enseignement, puis d'accéder à la profession enseignante.

L'utilisation du référentiel des compétences professionnelles est donc pertinente pour servir d'interface pour discuter de l'insertion professionnelle avec les nouveaux enseignants en ÉPS, et, par conséquent, pour répondre au but de l'étude, soit de décrire les perceptions de nouveaux enseignants en ÉPS à l'égard de leurs compétences professionnelles. Ce référentiel comporte douze compétences professionnelles réparties en quatre catégories décrites brièvement.

14 La première catégorie « Fondements » regroupe deux compétences :

- La CP1 « Agir en tant que professionnelle ou professionnel héritier, critique et interprète d'objets de savoirs ou de culture dans l'exercice de ses fonctions » a trait à la maitrise du programme de formation en ÉPS, de même qu'aux connaissances permettant d'aider les élèves à développer les compétences disciplinaires de ce programme. La maîtrise de la CP1 comprend aussi la capacité à juger de la valeur des nouvelles connaissances disponibles pour l'enseignement de l'ÉPS ;

- La CP2 « Communiquer clairement et correctement dans la langue d'enseignement, à l'oral et à l'écrit, dans les divers contextes liés à la profession enseignante » a trait aux capacités de l'enseignant d'écrire et de s'exprimer dans un langage qui convient à ce que la société attend d'un professionnel de l'enseignement. Au terme de sa formation, il est attendu qu'il utilise correctement les règles de la langue française lors de ses communications orales et écrites, qu'il puisse corriger les erreurs des élèves principalement lorsqu'ils s'expriment oralement, qu'il utilise un vocabulaire essentiellement en français, spécifique aux activités physiques et sportives et qu'il adapte son niveau de langage aux personnes à qui il s'adresse. La deuxième catégorie, "Acte d'enseigner", comprend quatre compétences qui mettent en évidence la complexité des diverses interactions entre l'enseignant et les élèves.

- La CP3 « Concevoir des situations d'enseignement-apprentissage pour les contenus à faire apprendre, et ce, en fonction des élèves concernés et du développement des compétences visées dans le programme de formation » a trait aux capacités de l'enseignant à élaborer, concevoir et planifier des situations d'enseignement-apprentissage en ÉPS. L'enseignant en ÉPS devra savoir organiser les séquences d'enseignement, programmer les activités et faire des liens entre les activités proposées aux élèves tout en tenant compte des caractéristiques affectives et physiques des élèves à qui il enseigne afin d'assurer une progression de leurs apprentissages ; 
- La CP4 «Piloter des situations d'enseignement-apprentissage pour les contenus à faire apprendre, et ce, en fonction des élèves concernés et du développement des compétences visées dans le programme de formation » a trait à ce qui se passe lors des interactions entre l'élève et l'enseignant et qui a pour but le développement des compétences disciplinaires de l'élève. Afin de motiver les élèves à apprendre, il est attendu de l'enseignant en ÉPS qu'il puisse démontrer la pertinence des activités proposées et qu'il aide les élèves à activer leurs acquis antérieurs pour apprendre. Il est aussi attendu que l'enseignant en ÉPS utilise le questionnement pour guider les élèves et qu'il les invite fréquemment à s'entraider pour favoriser leurs apprentissages ;

- La CP5 «Évaluer la progression des apprentissages et le degré d'acquisition des compétences des élèves pour les contenus à faire apprendre » a trait à l'évaluation du développement des compétences des élèves. L'enseignant en ÉPS sera capable, entre autres, de recourir à des outils pour évaluer les compétences des élèves, de mettre en place un processus d'évaluation incluant l'utilisation de l'auto-évaluation des élèves et d'expliquer aux parents la note qui apparait au bulletin ;

- La CP6 « Planifier, organiser et superviser le mode de fonctionnement du groupe classe en vue de favoriser l'apprentissage et la socialisation des élèves » a trait à la gestion des élèves au gymnase. Ainsi, l'enseignant en ÉPS sait élaborer un système de fonctionnement efficace au gymnase, communiquer clairement aux élèves ses attentes au regard de ce système de fonctionnement, reconnaître rapidement les comportements inappropriés, appliquer les conséquences prévues lorsqu'un comportement inapproprié le justifie et obtenir la collaboration des élèves afin d'enseigner dans un climat agréable.

La troisième catégorie de compétence, «Contexte scolaire et social », regroupe les CP7 à CP10.

- La CP7 «Adapter ses interventions aux besoins et aux caractéristiques des élèves présentant des difficultés d'apprentissage, d'adaptation ou un handicap » a trait aux ajustements que l'enseignant doit faire pour adapter son enseignement aux besoins particuliers des élèves. Selon les attentes de formation envers l'enseignant en ÉPS, il doit posséder une connaissance suffisante des caractéristiques de fonctionnement des élèves à besoins particuliers, veiller à ce qu'ils soient accueillis par les autres élèves et jouer un rôle pertinent dans la mise en place des plans d'intervention pour ces élèves à besoins particuliers ;

- La CP8 « Intégrer les technologies de l'information et des communications aux fins de préparation et de pilotage d'activités d'enseignement-apprentissage, de gestion de l'enseignement et de développement professionnel » a trait à l'utilisation des technologies dans divers contextes. L'enseignant en ÉPS devra maîtriser les technologies de l'information et des communications utiles à l'accomplissement de ses tâches d'enseignant, les utiliser pour son développement professionnel continu et aider les élèves à les utiliser à des fins d'apprentissage ;

- La CP9 « Coopérer avec l'équipe école, les parents, les différents partenaires sociaux et les élèves en vue de l'atteinte des objectifs éducatifs de l'école » a trait au partenariat hors du lieu d'enseignement de l'ÉPS et porte sur les collaborations avec différents intervenants ayant pour but d'aborder des thématiques plus larges que l'acquisition des compétences disciplinaires du programme de formation. Ainsi, l'enseignant en ÉPS pourra collaborer à l'élaboration de projets pour l'école avec des partenaires non-enseignants, situer son rôle par rapport à celui des autres intervenants non-enseignants pour favoriser une complémentarité respectueuse des compétences professionnelles de chacun, être à l'aise 
pour communiquer avec les parents des élèves et soutenir les élèves lorsqu'ils collaborent aux projets de l'école ;

- La CP10 « Travailler de concert avec les membres de l'équipe pédagogique à la réalisation des tâches permettant le développement et l'évaluation des compétences visées dans le programme de formation, et ce, en fonction des élèves concernés » a trait à la collaboration entre les intervenants qui œuvrent à l'école en lien avec les compétences disciplinaires de l'ÉPS. L'enseignant en ÉPS sera en mesure de mener des projets pédagogiques en collaboration avec d'autres enseignants de l'école, participer avec d'autres enseignants à la planification des interventions auprès des élèves, travailler à l'obtention d'un consensus au sein de l'équipe pédagogique et discerner les situations pédagogiques où la collaboration des collègues enseignants l'exige.

17 La quatrième catégorie de compétences "Identité professionnelle » regroupe deux compétences qui invitent l'enseignant à actualiser sa formation et à agir de façon éthique et professionnelle (CP11 et CP12).

- La CP11 «S'engager dans une démarche individuelle et collective de développement professionnel » a trait aux initiatives à prendre par les enseignants en ÉPS pour développer leur expertise. Les attentes de formation sont en lien avec l'établissement d'un bilan des compétences professionnelles, l'échange avec des collègues sur la pertinence des choix pédagogiques, l'analyse de ses pratiques professionnelles et l'utilisation des ressources disponibles pour les améliorer ;

- Finalement, la CP12 «Agir de façon éthique et responsable dans l'exercice de ses fonctions » amène l'enseignant en ÉPS à reconnaître les valeurs qu'il véhicule lorsqu'il enseigne, assumer la responsabilité de ses choix pédagogiques dans l'exercice de ses fonctions, agir de manière professionnelle et équitable avec tous les élèves.

18 C'est grâce à l'utilisation du référentiel des compétences que l'insertion professionnelle sera étudiée. Plus spécifiquement, les objectifs de cette étude sont d'identifier, au regard de la perception des nouveaux enseignants en ÉPS, les compétences jugées les plus et les moins déterminantes pour l'insertion professionnelle ainsi que les compétences qu'ils maîtrisent le plus et le moins. Une meilleure compréhension de l'insertion professionnelle au regard des compétences professionnelles permettra de développer des connaissances pertinentes quant à la formation initiale des enseignants d'ÉPS.

\section{Méthode}

Afin de permettre aux participants de porter à la fois un regard sur eux-mêmes (les compétences qu'ils maîtrisent) et sur les exigences du milieu professionnel (les compétences qu'ils jugent déterminantes), une approche qualitative interprétative a été privilégiée pour cette étude qui a reçu l'approbation du comité d'éthique de l'Université du Québec à Montréal.

\subsection{Les participants}

Au printemps 2011, au terme de leur formation en enseignement de l'ÉPS, seize finissants volontaires ( 7 femmes et 9 hommes) ont accepté de participer à l'étude. Selon leur choix personnel, ils se destinent soit à l'enseignement primaire $(n=7)$ ou à l'enseignement secondaire $(n=9)$. Ils sont âgés de 23 à 34 ans (moyenne $=26$ ans) et ils 
ont cumulé quelques expériences d'enseignement de l'ÉPS lors de leurs stages et des suppléances réalisées durant leur formation. L'approche qualitative interprétative privilégiée pour cette étude ne conduit cependant pas à l'analyse de différences selon les caractéristiques des participants.

\subsection{Outil}

D'une durée moyenne de 45 minutes, les entretiens individuels semi-dirigés ont permis d'approfondir les perceptions des nouveaux enseignants en ÉPS tout juste avant leur entrée dans la profession. Afin de joindre les participants facilement, peu importe leur région de résidence, le mode téléphonique a été utilisé et un canevas d'entretien a été élaboré à partir du questionnaire de Martineau et Presseau (2004). Quatre des quinze questions de ce canevas font l'objet du présent article :

1. Parmi les douze compétences professionnelles, lesquelles te semblent les plus déterminantes à ton début de carrière?

2. Parmi les douze compétences professionnelles, lesquelles te semblent les moins déterminantes à ton début de carrière?

3. Peux-tu me nommer quelques-unes des compétences professionnelles que tu crois le plus maîtriser?

4. Peux-tu me nommer quelques-unes des compétences professionnelles que tu crois le moins maîtriser?

Lors des entretiens, qui se sont échelonnés au cours des mois de mai et juin 2011, les participants avaient en mains la liste des douze compétences professionnelles du référentiel.

\subsection{Stratégie d'analyse}

Chaque entrevue a été enregistrée puis transcrite intégralement afin de permettre l'analyse de contenu qui a fait appel à des catégories prédéterminées représentées par le référentiel des douze compétences professionnelles (MEQ, 2001). La stratégie d'analyse de contenu comporte quatre étapes adaptées de Boutin (2007) :

1. Lectures préliminaires des données et établissement de la liste d'énoncés;

2. Regroupement des énoncés selon les douze compétences professionnelles ;

3. Identification de sous-catégories de chacune des douze compétences professionnelles ;

4. Description des résultats.

Dans un premier temps, les 314 énoncés ont été classés par consensus par deux des cinq chercheurs de l'équipe. Dans un deuxième temps, deux autres personnes ont classé, de façon indépendante et à l'aveugle, un échantillon de 80 énoncés, représentant $25 \%$ du corpus, dont la répartition dans les catégories était identique à la répartition de l'ensemble des énoncés du corpus. Ces codeurs ont obtenu un taux d'accord de 93,6\% et $94,9 \%$.

\section{Résultats}

Les résultats permettent de décrire les perceptions de nouveaux enseignants en ÉPS à l'égard des compétences professionnelles qu'ils considèrent les plus déterminantes 
pour leur insertion professionnelle ainsi que celles qu'ils maîtrisent le mieux. Le sommaire des résultats (voir tableau 1) présente le nombre de participants ayant identifié les compétences professionnelles pour les quatre questions qui sont traitées ici.

Tableau 1 : Participants $(n=16)$ ayant nommé les compétences professionnelles comme étant plus ou moins déterminantes et maîtrisées

\begin{tabular}{|c|c|c|c|c|c|}
\hline \multirow{2}{*}{\multicolumn{2}{|c|}{ Compétences professionnelles (MEQ, 2001) }} & \multicolumn{2}{|c|}{ Déterminantes } & \multicolumn{2}{|c|}{ Maîtrisées } \\
\hline & & Plus & Moins & Plus & Moins \\
\hline 1 & $\begin{array}{l}\text { Agir en tant que professionnelle ou professionnel héritier, } \\
\text { critique et interprète d'objets de savoirs ou de culture dans } \\
\text { l'exercice de ses fonctions }\end{array}$ & 2 & 0 & 6 & 0 \\
\hline 2 & $\begin{array}{l}\text { Communiquer clairement et correctement dans la langue } \\
\text { d'enseignement, à l'oral et à l'écrit, dans les divers contextes } \\
\text { liés à la profession enseignante }\end{array}$ & 6 & 0 & 10 & 1 \\
\hline 3 & $\begin{array}{l}\text { Concevoir des situations d'enseignement-apprentissage pour } \\
\text { les contenus à faire apprendre, et ce, en fonction des élèves } \\
\text { concemés et du développement des competences visées dans le } \\
\text { programme de formation }\end{array}$ & 10 & 3 & 6 & 2 \\
\hline 4 & $\begin{array}{l}\text { Piloter des situations d'enseignement-apprentissage pour les } \\
\text { contenus à faire apprendre, et ce, en fonction des élèves } \\
\text { concemés et du développement des compétences visées dans le } \\
\text { programme de formation. }\end{array}$ & 11 & 1 & 8 & 1 \\
\hline 5 & $\begin{array}{l}\text { Evaluer la progression des apprentissages et le degré } \\
\text { d'acquisition des compétences des éleves pour les contenus à } \\
\text { faire apprendre }\end{array}$ & 7 & 3 & 2 & 11 \\
\hline 6 & $\begin{array}{l}\text { Planifier, organiser et superviser le mode de fonctionnement du } \\
\text { groupe-classe en vue de favoriserl'apprentissage et la } \\
\text { socialisation des élèves }\end{array}$ & 6 & 1 & 5 & 2 \\
\hline 7 & $\begin{array}{l}\text { Adapter ses interventions aux besoins et aux caractéristiques } \\
\text { des élèves présentant des difficultés d'apprentissage, } \\
\text { d'adaptationou un handicap }\end{array}$ & 0 & 3 & 3 & 10 \\
\hline 8 & $\begin{array}{l}\text { Intégrer les technologies de l'information et des } \\
\text { communications aux fins de préparation et de pilotage } \\
\text { d'activités d'enseignement-apprentissage, de gestion de } \\
\text { l'enseignement et de développement professionnel }\end{array}$ & 0 & 12 & 2 & 4 \\
\hline 9 & $\begin{array}{l}\text { Cooperer avecl'équipe-école, les parents, les différents } \\
\text { partenaires sociaux et les élèves en vue del'atteinte des } \\
\text { objectifs éducatifs del'école }\end{array}$ & 6 & 1 & 6 & 4 \\
\hline 10 & $\begin{array}{l}\text { Travailler de concert avec les membres del'équipe } \\
\text { pédagogique à la réalisation des tâches permettant le } \\
\text { développement et l'évaluation des compétences visées dans le } \\
\text { programme de formation, et ce, en fonction des élèves } \\
\text { concemés }\end{array}$ & 2 & 1 & 4 & 1 \\
\hline 11 & $\begin{array}{l}\text { S'engager dans une démarche individuelle et collective de } \\
\text { développement professionnel }\end{array}$ & 3 & 2 & 6 & 1 \\
\hline 12 & $\begin{array}{l}\text { Aggir de façon éthique et responsable dansl'exercice de ses } \\
\text { fonctions }\end{array}$ & 5 & 0 & 9 & 0 \\
\hline
\end{tabular}

\subsection{Compétences les plus déterminantes et les moins déterminantes}

26 À la première question concernant les compétences professionnelles les plus déterminantes au début de carrière, les nouveaux enseignants en ÉPS évoquent principalement des compétences associées à la catégorie "Acte d'enseigner ", en particulier les CP4 et CP3 qui se partagent le premier rang. La CP4 est abordée par onze des seize participants, dont voici deux passages éloquents :

«Piloter, bien c'est certain que pour réussir à être un bon enseignant, selon moi il faut être capable de rendre la marchandise comme il le faut. Que ce soit en matière d'éducatifs intéressants, de situations d'apprentissage intéressantes, même dans la qualité de l'enseignement, je pense que ça irait aussi dans le pilotage " (Participant $\mathrm{N}^{\circ} 13$ ).

"Je crois qu'en début de carrière, on est beaucoup centré sur son enseignement parce que c'est un peu de là que tout part dans le fond »(Participant $\left.\mathrm{N}^{\circ} 4\right)$.

En ce qui a trait à la CP3 énoncée également par une majorité de participants (10/16), elle se résume ainsi :

"Parce que je pense que quand je vais commencer ma carrière en tant qu'éducateur physique, c'est vraiment là-dessus que je vais tenter de travailler, de me construire des situations d'apprentissage et d'évaluation pour les cours que je vais avoir à donner" (Participant $\mathrm{N}^{\circ} 7$ ). 
"C'est important de savoir concevoir parce que si on construit des situations d'enseignement-apprentissage, on va arriver plus préparés à nos cours puis les élèves vont en bénéficier, je pense qu'en début de carrière, c'est important parce qu'on arrive avec un certain bagage qu'on a eu dans notre baccalauréat, mais il reste quand même beaucoup de choses à construire [...] c'est plutôt important et au fil des ans, je vais en avoir moins à planifier qu'en début de carrière " (Participant $\mathrm{N}^{\circ}$ 9).

Les participants $(n=7)$, abordent la $\mathrm{CP} 5$, comme en témoigne ce passage qui résume assez bien l'ensemble des propos :

«La CP5, c'est comme la ligne directrice, c'est ce que le Ministère nous demande, ce que les parents veulent, ce que les jeunes veulent aussi donc il faut tout monter en lien avec l'évaluation. Je trouve que c'est vraiment important de bien la maîtriser, tout doit être en lien avec ça. Si ton évaluation est moche [mauvaise], tu as beau être un bon enseignant, mais les élèves vont quand même avoir de la difficulté à te prendre au sérieux si jamais ton évaluation ne tient pas debout [n'est pas pertinente] 》(Participant $\mathrm{N}^{\circ} 13$ ).

Dans une moindre mesure, ils ont identifié les CP2, CP6, CP9 et CP12. À titre d'exemple, les participants citent l'importance de la compétence langagière (CP2) de cette façon :

« La communication c'est toujours important, on doit adapter notre langage à la clientèle, soit au primaire ou au secondaire, et soit en sixième année ou en maternelle, c'est sûr que je n'utiliserais pas le même langage, donc c'est important d'être capable de transmettre l'information souhaitée » (Participant $\mathrm{N}^{\circ}$ 5).

Quelques-uns évoquent les $\mathrm{CP} 1, \mathrm{CP} 10$ et $\mathrm{CP} 11$, comme ce passage qui renvoie à la $\mathrm{CP} 10$ :

"Au début c'est encore plus important parce que tu n'as aucune preuve de faite, il faut que tu fasses tes preuves un peu aussi, la compétence dix dans le même ordre d'idée, s'intégrer dans l'équipe-école et puis travailler avec les gens avec qui on a à travailler pour peut-être plusieurs années » (Participant $\left.\mathrm{N}^{\circ} 6\right)$.

31 Seules les CP7 et CP8 n'ont été abordées par aucun participant lors de cette question qui visait à identifier les compétences les plus déterminantes en début de carrière.

32 À la seconde question évoquant les compétences les moins déterminantes au début de carrière, les participants soulèvent surtout la CP8 associée aux technologies de l'information et des communications :

«Peut-être intégrer les technologies de l'information [...] au début d'une carrière, comme moi je vais me promener d'une école à l'autre, c'est plus difficile d'avoir certains moyens puis de prendre le temps de développer des outils en lien avec les nouvelles technologies. Ça peut se faire, mais pour moi ce n'est pas une priorité " (Participant $\mathrm{N}^{\circ} 12$ ).

Aussi, la CP7 qui n’a pas été nommée lors de la question $\mathrm{N}^{\circ} 1$ a été nommée par trois participants, un de ceux-ci indique :

"Ça dépend des groupes que tu as au début, mais moi pour l'instant, ça ne me touche pas, j'ai personne en motricité réduite ou en difficulté grave d'apprentissage. Donc pour moi, ça ne me touche pas en début de carrière. C'est sûr qu'éventuellement ça va être le cas, il va falloir que je trouve d'autres méthodes de travail pour m'adapter à eux» (Participant $\mathrm{N}^{\circ} 11$ ).

34 De plus, trois participants ont fait référence aux $\mathrm{CP} 3$ et $\mathrm{CP} 5$ respectivement de la façon suivante :

"Concevoir des situations d'apprentissage pour quelqu'un qui fait beaucoup de suppléance, on y touche moins [...] même dans un remplacement d'un mois, il faut que je travaille avec la planification que l'enseignant a faite. Il faut que je m'inscrive dans sa façon de faire, parce que les élèves ont connu ça tout le long de l'année, et quand je vais partir ce sera encore ça qu'ils vont connaître, donc je ne peux pas arriver et tout chambarder [modifier]» (Participant $\mathrm{N}^{\circ} 2$ ). 
"C'est juste une fois qu'on est mieux installé dans l'école, une fois qu'on arrive à faire nos cours comme il faut, qu'on s'est adapté, qu'on est peut-être plus en mesure d'évaluer correctement » (Participant $\left.\mathrm{N}^{\circ} 6\right)$.

Pour leur part, les $\mathrm{CP} 4, \mathrm{CP} 6, \mathrm{CP} 9$ et $\mathrm{CP} 10$ ont chacune été proposées par un seul participant alors que les $\mathrm{CP} 1, \mathrm{CP} 2$ et $\mathrm{CP} 12$ n'ont pas été abordées en lien avec cette question.

\subsection{Compétences les plus maîtrisées et les moins maîtrisées}

En lien avec la troisième question, voici quelques énoncés se rattachant aux compétences professionnelles les plus maîtrisées par les futurs enseignants en ÉPS, soit respectivement les $\mathrm{CP} 2, \mathrm{CP} 12$ et $\mathrm{CP} 4$. Concernant la $\mathrm{CP} 2$ mentionnée par dix des seize participants :

"Je pense que je suis capable de bien m'exprimer, on avait des tests à faire en début de notre formation au niveau de l'expression orale, j'avais passé ça haut la main, le test de français aussi, je l'ai passé du premier coup » (Participant $\left.\mathrm{N}^{\circ} 10\right)$.

Pour sa part, la CP12 (9/16 participants), liée à l'éthique, est discutée de la façon suivante :

"Je crois que je la maîtrise, si je ne faisais pas ça, je n'aurais pas réussi mes stages" (Participant $\mathrm{N}^{\circ} 7$ ).

Quant au pilotage lié à la CP4, (8/16 participants) :

"C'est l'engagement envers les élèves et leurs apprentissages, je pense que c'est la fonction première de l'enseignant [...] les élèves me le disent beaucoup et mes collègues, peu importe que ce soit au niveau universitaire où j'ai enseigné, primaire ou secondaire, ce qu'on me dit : c'est l'fun à quel point on sent que tu es toujours présent, toujours en train de nous encourager, tu es debout, tu bouges sur le terrain, on sent que tu es là " (Participant $\left.\mathrm{N}^{\circ} 16\right)$. «Je la maîtrise très bien, je vous dirais. En tout cas je pense » (Participant $\left.\mathrm{N}^{\circ} 11\right)$.

Ensuite, six des seize participants en ÉPS soulèvent aussi d'autres compétences qu'ils estiment les plus maîtrisées, soit les CP1, CP3, CP9 et CP11. Par exemple en ce qui touche la CP9, les stages sont notamment abordés :

"Dans le cadre de mon dernier stage et dans les projets que j'ai faits dans mes stages, ça va toujours bien: travailler avec l'équipe-école, la direction, les autres enseignants, je m'implique bien, même que j'aime beaucoup ça m'impliquer dans des projets, donc ça, je pense que je l'ai facilement " (Participant $\left.\mathrm{N}^{\circ} 1\right)$.

La CP11 est discutée sous l'angle de l'ouverture à l'expérience des autres enseignants :

"Je pense que je suis quelqu'un qui est ouvert, accepte la critique donc j'ai avantage à aller voir différents points de vue, comment les autres travaillent. C'est sûr on a une division, des préjugés surtout envers les enseignants d'expérience, mais de voir ce qu'ils ont essayé, et ce qu'ils ont pas essayé, pourquoi ils ont changé tel ou tel aspect de leur enseignement, est-ce qu'il y a des choses qu'ils trouvent que ça va mieux avec certains types d'élèves, je pense qu'il y a énormément de savoirs à aller chercher là, alors ces personnes sont là pour ça [...] on a sans aucun doute avantage à aller explorer les connaissances des autres enseignants avec qui on travaille » (Participant $\left.\mathrm{N}^{\circ} 8\right)$.

41 Les CP5, CP6, CP7, CP8, et CP10 sont moins souvent citées comme étant les plus maîtrisées; les trois dernières étant toutes regroupées dans la catégorie "Contexte social et scolaire ».

Enfin, lors des réponses à la quatrième question relative aux compétences les moins maitrisées, deux compétences se démarquent, à savoir les CP5 (11/16 participants) et 
CP7 (10/16 participants). En regard de la CP5, les propos concernent notamment la formation initiale :

«Je trouvais qu'au travers de toute ma formation, une chose qui me manquait où je n'avais pas beaucoup d'outils, c'était sur l'évaluation » (Participant $\left.\mathrm{N}^{\circ} 16\right)$.

"Je trouve d'ailleurs que c'est quelque chose qui a manqué dans notre baccalauréat [...] il manquait certaines précisions, on était un petit peu laissé à nous-même au niveau de l'évaluation » (Participant $\left.\mathrm{N}^{\circ} 1\right)$. langue parlée et écrite, et des technologies de l'information et des communications, celles regroupées sous l'Acte d'enseigner revêtent sans contredit une importance majeure quant à l'insertion professionnelle des futurs enseignants en ÉPS interrogés au cours de cette étude. Cet intérêt pour des éléments relatifs au niveau professionnel (Vonk, 1995) confirme les récentes études qui proposent que les principales difficultés rencontrées sont d'ordre professionnel (Hill \& Brodin, 2004 ; Roux-Perez, 2003).

\subsection{Compétences les plus déterminantes et les moins déterminantes}

48 Le regard qu'ils portent sur les exigences du milieu professionnel, confirme les résultats d'études antérieures (Eldar, Nable, Schechter, \& Marzin, 2003; Macdonald, 1993 ; Mohr \& Townsend, 2001 ; Spallanzani, Desbien, \& Beaudoin, 2012), les nouveaux 
enseignants interrogés considèrent que les interactions vécues en cours d'ÉPS, selon la catégorie de "Acted'enseigner", sont les plus déterminantes pour leur insertion professionnelle. Conformément au renouveau pédagogique et aux orientations qui en découlent (MEQ, 2001), ils associent étroitement le processus d'enseignementapprentissage au développement des compétences des élèves. De fait, les propos qu'ils tiennent rendent justice à la cohérence qui doit exister dans les processus d'enseignement-apprentissage, soit la planification (CP3), l'enseignement (CP4) et l'évaluation (CP5). On remarque aussi que leurs propos sur l'importance des compétences de l'« Acte d'enseigner " sont fréquemment associés à ce que les élèves vont penser de leur enseignement. Ce sont là des résultats similaires à ceux des participants de l'étude de Shoval, Erlich, \& Fejgin (2009) qui soulignent que les nouveaux enseignants en ÉPS sont très soucieux de la perception qu'ont les élèves de leur prestation. Aussi, tel que l'ont souligné Spallanzani, Desbien et Beaudoin (2012) les nouveaux enseignants démontrent leur souci de mieux connaître leurs élèves, les soutenir, les guider et les accompagner non seulement dans leur cheminement scolaire, mais aussi dans leur cheminement personnel et social. En lien avec la catégorie "Fondements », ils jugent la CP2 liée à la communication orale et écrite déterminante en évoquant la nécessité de s'ajuster aux différents groupes et niveaux scolaires auxquels ils enseignent, ce qui correspond au contexte québécois des spécialistes, comme ceux en ÉPS, qui reçoivent une formation pour enseigner tant aux enfants du primaire (6-12 ans) qu'aux adolescents du secondaire (12-17 ans). Leurs propos révèlent leur souci de bien communiquer, et ce dans la perspective d'être appréciés et en harmonie avec leurs élèves, faisant une fois de plus écho aux travaux de Shoval, Erlich, \& Fejgin (2009).

49 À l'opposé et de façon univoque, l'utilisation des technologies de l'information et des communications (TIC) à des fins d'enseignement (CP8) les préoccupe peu puisqu'elle est jugée moins déterminante par 12 des 16 participants interrogés. À l'issue des entretiens, les participants estiment cette compétence est moins déterminante surtout parce que les milieux scolaires ne fournissent généralement pas le matériel ou les ressources nécessaires pour exploiter les TIC à des fins d'enseignement. Cette situation peut être spécifique à la discipline de l'ÉPS. Il est possible que les dépenses accordées aux infrastructures et au matériel sportif inhérent à l'ÉPS soit déjà très importantes laissant ainsi en plan des dépenses moins prioritaires comme les TIC. Tout comme Hebert et Worthy (2001) l'ont souligné, les caractéristiques du milieu d'accueil sont susceptibles d'influencer le succès de l'enseignant, mais aussi la perception de ses propres performances comme enseignant. Des études futures pourraient examiner de façon plus approfondie l'utilisation des technologies en ÉPS dans divers contextes et milieux scolaires afin de suggérer des pistes d'action visant à faciliter le déploiement de cette compétence chez les nouveaux enseignants de la discipline. La CP8 jugée moins déterminante soulève un autre élément de discussion. À l'instar de Meunier (2008), les enseignants en ÉPS sont pourtant ceux qui apprécient le plus l'utilisation de l'ordinateur de poche pour supporter leurs observations associées à l'évaluation. Cette seule étude ne nous permet pas de conclure à une contradiction avec nos résultats puisque nous nous adressons à des finissants et non pas à des enseignants en exercice. Il laisse néanmoins présager la nécessité de documenter les contenus de formation en ÉPS sur les TIC et leur utilisation dans le domaine de l'évaluation, et leur véritable transfert par les nouveaux enseignants en milieu professionnel. Afin de favoriser l'utilisation des TIC en ÉPS et en guise de nouvelle voie de recherche à explorer, nous 
partageons l'avis de certains chercheurs (Holzinger, Dorner, Manuela, Valdez, \& Ziefle, 2010 ; Kreps \& Neuhauser, 2010) concernant l'utilisation judicieuse de réseaux sociaux (Facebook, Youtube, Twitter, etc.) et des nouvelles technologies (GPS, téléphone intelligent, compteurs de pas) apparaît comme une façon renouvelée de rejoindre et d'engager les jeunes vers l'adoption de saines habitudes de vie, une visée de l'éducation à la santé en ÉPS du programme de formation de l'école québécoise.

\subsection{Compétences les plus maîtrisées et les moins maîtrisées}

Lorsqu'appelés à se prononcer sur leurs propres compétences, une forte majorité des participants affirme bien maîtriser la CP2 (compétence langagière). Dans un contexte où la qualité du français écrit des enseignants en formation est fortement décriée (Dion-Viens, 2010), il est pour le moins surprenant de constater qu'ils estiment maîtriser cette compétence. Deux hypothèses peuvent être émises. D'abord, ils ont surtout évoqué la qualité du français oral pour justifier leur maîtrise de cette compétence tel qu'un vocabulaire clair et varié, sans exposer véritablement la complexité de la langue écrite. De plus, plusieurs s'appuient sur la réussite d'un test de français écrit, une épreuve obligatoire pour l'obtention du permis d'enseigner au Québec, ce qui engendre peut-être un faux sentiment de compétence. D'un autre côté, certains participants ont associé la maîtrise de la CP2 aux modalités de communication facilitant la transmission d'information aux élèves et la relation enseignant-élève ce qui laisse entrevoir une interprétation différente de la CP2 entre les formateurs universitaires et les étudiants. La CP12 qui traite de l'éthique professionnelle semble être aussi une compétence maîtrisée pour la plupart des participants. Ils affirment ne pas avoir de problèmes avec l'éthique professionnelle. Bien que les écrits sur l'insertion professionnelle n'abordent pas directement cette compétence, l'étude de Martineau et Presseau (2003) rappelle que les directions d'école accordent une importance majeure à l'éthique professionnelle des nouveaux enseignants. De plus, dans de nombreux programmes de formation universitaire, cette compétence est dite éliminatoire lors des stages en ce sens qu'un manquement à l'éthique professionnelle peut mener directement à un échec au stage. Plusieurs participants admettent aussi maîtriser les $\mathrm{CP} 3$ et CP4, qui font partie de l'« Acte d'enseigner ». Certains s'appuient sur une forme de reconnaissance venant des élèves ou des pairs, à savoir les autres stagiaires et les enseignants associés qui les ont accueillis lors des stages, ce qui renforce l'importance d'un milieu de stage stimulant pour le stagiaire en ÉPS. Tel que le soulignent plusieurs auteurs (Blankenship \& Coleman, 2009; Curtner-Smith, 1998), la qualité du milieu de stage est primordiale pour l'insertion professionnelle future de l'enseignant. Ces auteurs insistent aussi sur l'importance d'offrir un milieu de stage qui présente des similitudes avec le milieu où l'enseignant débutera sa carrière. Il pourra alors développer ses compétences professionnelles dans un contexte similaire à celui du début de sa carrière.

En ce qui a trait aux compétences moins bien maitrisées, les CP5 et CP7 ressortent de façon très convaincante. Ainsi, la $\mathrm{CP} 5$ qui traite de l'évaluation des élèves semble aux yeux des nouveaux enseignants une de leurs faiblesses principales. C'est aussi ce qu'ont déclaré plusieurs participants américains à l'étude de Hill et Brodin (2004), car 71,6\% d'entre eux considèrent qu'il est " extrêmement difficile » à " modérément difficile " d'évaluer les élèves lors de leur première année d'enseignement, et ce malgré que $78,1 \%$ des 132 participants estiment que l'évaluation des élèves ait été vue lors de leur 
formation. Ceci renvoie peut-être à un certain décalage entre les contenus de formation, dans ce cas-ci en évaluation, et les véritables besoins sur le terrain, tout comme l'ont déjà souligné certains chercheurs en considérant un décalage théorie vs pratique (Mohr \& Townsend, 2001 ; Roux-Perez, 2003). En ce qui a trait à la CP7, qui s'intéresse à l'adaptation de l'enseignement aux besoins particuliers des élèves, les perceptions de nos participants sont très semblables aux enseignants en ÉPS américains (Hill \& Brodin, 2004) et israéliens (Fejgin, Talmor, \& Erlich, 2005). En effet, Hill et Brodin (2004) soulignent que cette difficulté se classe au $3^{\mathrm{e}}$ rang des difficultés les plus importantes vécues lors de la première année d'enseignement. Pour sa part, Fejgin, Talmor et Erlich (2005) indiquent que les enseignants en ÉPS estiment que les mauvaises conditions dans lesquelles ils doivent favoriser l'inclusion des élèves présentant des besoins particuliers sont une source importante de stress. Ils indiquent que les installations et le matériel didactique ne sont pas adaptés, qu'ils doivent enseigner à de nombreux élèves en attente de diagnostic et de traitement, ce qui les empêche de modifier adéquatement leur enseignement. De plus, ils indiquent ne pas trop savoir comment adapter l'évaluation pour répondre aux besoins de ces élèves et expliquer les notes aux parents. Enfin, Huot (2008) rapporte que les éducateurs physiques québécois soulignent de sérieuses lacunes pour réussir une intégration efficace puisqu'ils ont rarement la possibilité de participer au plan d'intervention de ces élèves, qu'ils manquent de temps pour ajuster les planifications, le matériel et les évaluations et déplorent le manque de ressources matérielles, humaines et financières pour répondre correctement aux besoins de ces élèves. En résumé, les difficultés relevées par nos participants renvoient principalement au niveau professionnel suggéré par Vonk (1995), à savoir les connaissances et les pratiques enseignantes, tout comme l'ont rapporté Shoval, Erlich, \& Fejgin (2009). À notre avis, il n'est pas étonnant que les niveaux personnel et environnemental soient peu retenus dans les perceptions des participants puisque les fondements entourant le référentiel québécois des douze compétences professionnelles qui ont servi de point de départ à l'entretien, sont justement axés sur le développement professionnel, donc inclus dans le niveau professionnel de Vonk (1995).

\subsection{Une perception de maîtrise des compétences professionnelles à questionner}

52 À titre de compétences bien maîtrisées, plusieurs participants ont identifié les compétences associées à la maîtrise de la langue (CP2), au pilotage des situations d'enseignement-apprentissage (CP4) et à l'éthique professionnelle (CP12). Ces résultats sont similaires à ceux de Monfette, Grenier et Gosselin (2012) qui ont mesuré le sentiment d'efficacité personnelle des stagiaires de la première à la quatrième année et qui obtiennent un score très élevé à ces trois compétences, les classant parmi les quatre plus importantes. Toutefois, il nous semble inquiétant de constater que deux des compétences considérées comme des forces par les participants (CP2 et CP12) ne figurent pas parmi les compétences les plus déterminantes pour leur insertion professionnelle. Ainsi, l'adéquation entre leurs forces et leur perception des exigences du milieu scolaire semble plutôt discordante. Il est possible aussi que les participants à l'étude perçoivent ces deux compétences de manière plutôt transversale par rapport aux compétences de l'Acte d'enseigner et n'en jugent pas autant l'importance. De plus, alors que onze participants indiquent que la compétence liée à l'évaluation des élèves 
(CP5) est peu maîtrisée cinq d'entre eux estiment qu'elle est parmi les plus déterminantes à l'insertion professionnelle. Une discordance cette fois qui relève d'une faiblesse chez les participants pourtant jugée déterminante en phase d'insertion professionnelle. Est-ce là une information importante à prendre en compte dans la formation initiale? Si les étudiants finissant de notre étude semblent douter de leur capacité à réaliser une tâche qu'ils jugent pourtant très importante, n'est-ce pas un signal qu'il faille mettre plus d'accent sur le développement de cette compétence? D'ailleurs, les récentes études démontrent clairement que l'évaluation des élèves constituent des difficultés majeures pour plusieurs enseignants en début de carrière (Hill \& Brodin, 2004 ; Fejgin, Talmor, \& Erlich, 2005).

\subsection{Une perception du milieu professionnel à ajuster}

53 Le regard posé par les participants sur leurs compétences déterminantes et maîtrisées permet de constater que leur perception du milieu professionnel devra nécessairement être recadrée, bien que certaines de leurs lectures soient justes. Parmi celles-ci, les participants à cette étude anticipent qu'il sera déterminant dans le milieu scolaire de savoir piloter des situations d'enseignement-apprentissage (CP4), de concevoir ces situations (CP3) et d'évaluer les élèves (CP5). Leur perception est assez semblable à la réalité décrite par les enseignants en ÉPS débutants interrogés par plusieurs auteurs (Mohr \& Townsend, 2001; Roux-Perez, 2003; Shoval, Erlich, \& Fejgin, 2009). Cette perception juste de ce qui les attend et leurs attentes réalistes pourrait potentiellement constituer des facteurs de protection importants pour surmonter les défis inhérents à l'insertion professionnelle qu'ils s'apprêtent à vivre (Leroux \& Théorêt, 2011). Par contre, il paraît étonnant que la gestion de classe (CP6) et la compétence associée à l'adaptation de l'enseignement aux élèves présentant des besoins particuliers (CP7) ne soient pas perçues par autant de participants comme des compétences aussi déterminantes pour une insertion professionnelle réussie. Sachant que la gestion de classe et l'adaptation de l'enseignement aux élèves présentant des besoins particuliers sont à la fois liées entre elles (Hill \& Brodin, 2004) et associées au risque d'épuisement professionnel relevé dans la littérature (Fejgin, Talmor, \& Erlich, 2005) ainsi qu'au choc de la réalité (Mohr \& Townsend, 2001; Roux-Perez, 2003), il apparaît primordial de trouver des moyens d'ajuster la formation des enseignants en ÉPS afin qu'ils débutent leur carrière avec une vision plus juste de ce qui les attend. Nous supposons ainsi que des attentes réalistes à l'égard de ces deux compétences (CP6 et CP7) pourraient prémunir les nouveaux enseignants de problèmes psychologiques tels qu'un sentiment de stress élevé (Cossette, 1999) ou qu'un épuisement professionnel (Fives, Hamman, \& Olivarez, 2007).

\section{Conclusion}

Cette étude avait pour but de décrire les perceptions de nouveaux enseignants en ÉPS à l'égard des compétences professionnelles qu'ils considèrent les plus déterminantes à leur insertion professionnelle ainsi que celles qu'ils maîtrisent le mieux, telles qu'édictées par le référentiel québécois ( $\mathrm{MEQ}, 2001)$. L'analyse des propos des 16 finissants recueillis lors d'entrevues individuelles semi-dirigées illustre quatre grands constats : 1) la planification (CP3) et le pilotage des situations d'enseignement (CP4) 
sont les compétences perçues comme étant les plus déterminantes pour l'insertion professionnelle ; 2) la capacité d'utiliser les technologies de l'information et de la communication semble peu déterminante ; 3 ) la communication avec les élèves (CP2) et les comportements d'ordre éthique professionnelle (CP12) sont les compétences les plus maîtrisées; et 4) l'évaluation des élèves (CP5) et l'adaptation de l'enseignement aux élèves présentant des besoins particuliers (CP7) sont les compétences professionnelles qu'ils maîtrisent le moins.

55 Cette étude présente des limites méthodologiques, notamment compte tenu d'un petit échantillon, d'une collecte ponctuelle de données et de l'utilisation du référentiel québécois de compétences professionnelles pour guider l'entretien. En contrepartie, le référentiel permet d'apporter un éclairage nouveau de l'insertion professionnelle en ÉPS et de la formation initiale qui y est associée en contexte québécois. Ainsi, nous croyons que la formation universitaire en enseignement doit poursuivre le développement des douze compétences professionnelles en favorisant davantage les compétences de l'Acte d'enseigner, dont une plus grande attention sur celle en lien avec l'évaluation des apprentissages qui serait susceptible de contribuer à un plus grand sentiment de maîtrise des futurs enseignants. De plus, des efforts semblent nécessaires afin que les enseignants en ÉPS puissent véritablement participer à l'intégration des élèves présentant des besoins particuliers, soit la CP7. D'ailleurs, tel que le suggèrent Knoblauch et Woolfolk Hoy (2008), la formation initiale doit permettre aux futurs enseignants de développer un sentiment d'efficacité personnelle suffisamment fort pour faire face aux différentes difficultés rencontrées par la suite dans leur milieu de travail. Plus spécifiquement selon Nichols (2011), la formation dite pratique comme les stages permet de construire la confiance du stagiaire en regard de plusieurs aspects dont les interventions auprès des élèves présentant des besoins particuliers et est susceptible de consolider cette confiance par rapport à l'insertion professionnelle.

Somme toute, cette étude invite à une réflexion entourant les défis mis à jour de l'insertion professionnelle en ÉPS et le rôle crucial de la formation initiale dans sa mission de développer les douze compétences professionnelles chez tous les futurs enseignants. Des recherches futures portant sur des comportements ou attitudes observables par d'autres acteurs impliqués seraient toutes indiquées.En guise d'autres pistes de recherche, un suivi de l'évolution des perceptions des enseignants en ÉPS en début de carrière, et ce, à travers leur parcours d'insertion pendant les cinq premières années de pratique, permettrait de contribuer à une compréhension plus approfondie de ce phénomène dans le domaine spécifique de l'ÉPS. Par ailleurs, une étude menée de façon longitudinale permettrait de voir apparaître, si tel est le cas, la régression de leur sentiment de compétences (Shoval, Erlich, \& Fejgin, 2010). De récentes études utilisant le concept de résilience des enseignants (Leroux \& Théorêt, 2011) nous apparaissent aussi représenter une piste pertinente pour mettre en relation divers aspects inhérents à l'insertion professionnelle des enseignants débutants en ÉPS. En effet, le concept de la résilience chez les enseignants suscite l'intérêt récent d'un nombre grandissant de chercheurs (Le Cornu, 2009; Mansfield, Beltman, Price, \& McConney, 2012), car il permet de s'intéresser à l'adaptation positive en contexte d'adversité tel que celui de l'insertion professionnelle, ce qui semble être une avenue prometteuse pour contrer les obstacles d'une insertion qui demeure difficile suite à la transformation de la pratique enseignante observée depuis une dizaine d'années au Québec. Non explorée à ce jour en ÉPS, la voie de la résilience constitue une piste originale pour examiner l'ÉPS en 
particulier et ses nouveaux défis professionnels reliés notamment à l'éducation à la santé.

\section{BIBLIOGRAPHIE}

Blankenship, T. B., \& Coleman, M. (2009). An examination of « waxh-out » and workplace conditions of beginning physical education teachers. Physical Educator, 2, 97-111.

Boutin, G, (2007). L'entretien de groupe en recherche et formation. Montréal : Éditions nouvelles.

Comité d'orientation sur la formation du personnel enseignant (COFPE). (2002). Offrir la profession en héritage: Avis du COFPE sur l'insertion dans l'enseignement. Québec : Gouvernement du Québec.

Conseil supérieur de l'éducation (CSE). (2004). Un souffle nouveau pour la profession enseignante : avis au ministre de l'Éducation. Québec : Bibliothèque nationale du Québec.

Cossette, F. (1999). Les sources de stress, le stress ressenti et le soutien social chez les enseignants en début de carrière. Mémoire de maîtrise non publié, Université du Québec à Montréal/ Université du Québec à Trois-Rivières, Montréal/Trois-Rivières (Canada).

Curtner-Smith, M.D. (1998). Influence of biography, teacher education, and entry into the workforce on the perspectives and practices of first-year elementary school physical education teachers. European Journal of Physical Education, 3(1), 75-98.

Curtner-Smith, M.D. (2001). The occupational socialization of a first-year physical education teacher with a teaching orientation. Sport Education and Society, 6(1), 81-105.

Dion-Viens, D. (2010). Courchesne refuse de réduire les exigences en français pour les futurs profs. Repéré à www.cyberpresse.ca/le-soleil/actualites/education/201002/22/01-954291-courchesnerefuse-de-reduire-les-exigences-en-francais-pour-les-futurs-profs.php.

Eldar, E., Nable N., Schechter, C.T., \& Marzin, K. (2003). Anatomy of success and failure : the story of three novice teachers. Educational Research, 1, 29-48.

Fantilli, R., \& McDougall, D.E. (2009). A study of novice teachers : Challenges and supports in the first years. Teaching and Teacher Education, 25(6), 825-841.

Feiman-Nemser, S., Schwille, S., Carver, C., \& Yusko, B. (1999). A Conceptual Review of Litterature on New Teacher Induction. Washington, DC : National Partnership for Excellence and Accountability in Teaching.

Fejgin, N., Talmor, R., \& Erlich, I. (2005). Inclusion and burnout in physical education. European Physical Education Review, 11(1), 29-50.

Fives, H., Hamman, D., \& Olivarez, A. (2007). Does burnout begin with student-teaching ? Analyzing efficacy, burnout, and support during the student-teaching semester. Teacher and Teaching Education, 23, 916-936.

Hand, K.E. (2007). An examination of the induction experiences of beginning physical education teachers regarding their self-perceptions of teaching efficacy and the role of effectiveness of their mentor. Thèse de doctorat non publiée, Northern Illinois University, DeKalb. 
Hebert, E., \& Worthy, T. (2001). Does the first year of teaching have to be a bad one? A case study of success. Teaching and teacher education, 17, 897-911.

Hill, G., \& Brodin, K.L. (2004). Physical education teachers' perceptions of the adequacy of university coursework in preparation for teaching. Physical Educator, 61(2), 75-87.

Hoy, A. W., \& Spero, R. B. (2005). Changes in teacher efficacy during the early years of teaching : A comparison of four measures. Teaching and Teacher Education, 21, 343-356.

Holzinger, A., Dorner, S., Manuela, F., Valdez, A.C., \& Ziefle, M. (2010). Chances of Increasing Youth Health Awareness through Mobile Wellness Applications. Lecture Note in Computer Science, 6389, 71-81.

Huot, M.-A. (2008). Les attitudes des enseignants en éducation physique de niveau primaire face à la politique de l'adaptation scolaire et face à son application lorsqu'ils intègrent des ÉHDAA dans leurs groupes. Mémoire de maîtrise non publié, Université du Québec à Montréal, Montréal. Repéré à http://www.archipel.uqam.ca/1766/.

Knoblauch, D., \& Woolfolk Hoy, A. (2008). "Maybe I can teach those kids". The influence of contextual factors on student teacher's efficacy beliefs. Teaching and Teacher Education, 24(1), 166-179.

Kreps, G.L., \& Neuhauser, L. (2010). New Directions in eHealth communication : Opportunities and challenge. Patient Education and Counseling, 78, 329-336.

Lamarre, A. M. (2010). Donner un sens à l'expérience et à l'accompagnement. Vivre le primaire, 23(2), 41-43.

Le Cornu, R. (2009). Building resilience in pre-service teachers. Teaching and Teacher Education, 25, 717-723.

Le Maistre, C., \& Paré, A. (2010). Whatever it takes : How beginning teachers learn to survive. Teaching and Teacher Education,26, 559-564.

Leroux, M., \& Theorêt, M. (2011). Using the Critical Incident Technique to Frame Teacher Reflection : A Promising Approach to Foster Resilience.Communication présentée à la rencontre annuelle de American Educational Research Association, Nouvelles-Orléans.

Macdonald, D. (1993). Why do they leave ? Physical education teacher attrition. Communication présentée à la conference annuelle de Australian Association for Research in Education Annual Conference. Fremantle.

Macdonald, D. (1995). The role of proletarianization in physical education teacher. Research Quartely for Exercise and Sport, 66(2), 129-141.

Mansfield, C. F., Beltman, S., Price, A., \& McConney, A. (2012). "Don't sweat the small stuff" : Understanding teacher resilience at the chalkface. Teaching and Teacher Education, 28(3), 357-367.

Martineau, S., \& Presseau, A. (2003). Le sentiment d'incompétence pédagogique des enseignants en début de carrière et le soutien à l'insertion professionnelle. Brock Education, 2, 54-67.

Martineau, S., \& Presseau, A. (2004). Analyse exploratoire du discours sur la pratique chez des enseignants d'un CFER. Revue des sciences de l'éducation, 3, 631-646.

Meunier, H. (2008). Mise à l'essai de l'ordinateur de poche pour l'observation en classe dans le cadre d'une démarche d'évaluation des compétences. Mémoire de maîtrise non publié, Université du Québec à Montréal, Montréal. [M10228]. Repéré à http://virtuolien.uqam.ca/tout/ UQAM_BIB000048601. 
Ministère de l'Éducation du Québec (MEQ). (2001). La formation à l'enseignement, les orientations et les compétences professionnelles. Québec : Ministère de l'éducation.

Mohr, D., \& Townsend, S. (2001). In the beginning : New physical education teachers' quest for success. Teaching elementary physical education, 4, 9-13.

Monfette, O., Grenier, J., \& Gosselin, C. (2012). Influence des stages sur le sentiment d'efficacité personnelle des stagiaires. Actes de la $7^{\mathrm{e}}$ biennale de l'Association pour la recherche en intervention en sport (ARIS), Amiens, France.

Mukamurera, J. (2005). S'insérer dans l'enseignement au Québec : portrait des défis et enjeux pour la profession. Éducateur, 11, 29-31.

Mukamurera, J., Bourque, J., \& Ntebuste, J.G. (2010). La situation d'insertion professionnelle chez les enseignants du primaire au Québec. Vivre le primaire, 23(2), 36-37.

Nichols, B. (2011). Preservice Teachers' Perception of a Final Field Experience. Thèse de doctorat, Arkansas State University, Jonesboro.

Portelance, L., Mukamurera, J., Martineau, S., \& Gervais, C. (Éds.). (2008). L'insertion dans le milieu scolaire : une phase cruciale du développement professionnel de l'enseignant. Québec: Presses de l'Université Laval.

Roux-Perez, T. (2003). Processus identitaires dans la carrière des enseignants : deux études de cas en éducation physique et sportive (EPS). STAPS, 72(2), 35-47.

Solmon, M. A., Worthy, T., \& Carter, J.A. (1993) The Interaction of School Context and Role Identity of First-Year Teachers. Journal of Teaching in Physical Education, 12(3), 313-328.

Shoval, E., Erlich, I., \& Fejgin, N. (2010). Mapping and interpreting novice physical education teachers' self-perceptions of strengths and difficulties. Physical Education \& Sport Pedagogy, 14(1), 85-101.

Sirna, K., Tinning, R., \& Rossi, T. (2008). The social tasks of learning to become a physical education teacher : considering the HPE subject department as a community of practice. Sport, Education and Society, 13(3), 285-300.

Smyth, D. (1995) First-Year Physical Education Teachers' Perceptions of Their Workplace. Journal of Teaching in Physical Education, 14(2), 198-214.

Spallanzani, C., Desbiens, J-F., \& Beaudoin, S. (2012). Des perceptions d'éducateurs physiques quant à leur processus d'insertion socioprofessionnelle. Dans G. Carlier, C. Borges, M. Clerx et C. Delens (dir.), Identité professionnelle en éducation physique (p. 233-252). Louvain : Presses universitaires de Louvain.

Stroot, S.A. (2003). Organizational socialization : Factors impacting beginning teachers. Dans S. Silverman, \& C. Ennis (dir.), Student learning in physical education : applying research to enhance instruction (p. 339-366). Champaign : Human Kinetics.

Stroot, S.A., \& Ko, B. (2006). Teacher socialization and induction. Dans D. Kirk, M. O’Sullivan, \& D. Macdonald (dir.), Handbook of Research in Physical Education (p425-448). Berkeley, CA : Sage Publications.

Tait, M. (2008). Resilience as a contributor to novice teacher success, commitment, and retention. Teacher Education Quarterly, 35(4), 57-75.

Vonk, J.H.C. (1995). Conceptualizing novice teachers' professional development : A base for supervisory interventions. Communication présentée à la rencontre annuelle de la American Educational Research Association, San Francisco, CA. 
Wright, M.T. (2001). The use of ongoing, in-class assessment as a method of accountability during physical education. Thèse de doctorat, Oregon State University.

\section{RÉSUMÉS}

Le but de l'étude est de décrire les perceptions de nouveaux enseignants en éducation physique et à la santé (ÉPS) à l'égard de l'insertion professionnelle et de leurs compétences professionnelles. Au terme de leur formation initiale, seize finissants en ÉPS ont été interrogés lors d'entrevues individuelles semi-dirigées. Les résultats indiquent que les compétences reliées à la conception et au pilotage des situations d'enseignement seraient les plus déterminantes en début de carrière, alors que celle relative à l'utilisation des technologies est jugée moins déterminante. Interrogés également sur le niveau de maîtrise de leurs compétences, ils considèrent surtout être à l'aise pour communiquer clairement avec les élèves et agir de façon éthique. Cependant l'évaluation des élèves et l'adaptation de l'enseignement pour les élèves présentant des besoins particuliers semblent être moins bien maîtrisées. Certaines perceptions convergentes et divergentes sont discutées à la lumière d'une insertion professionnelle réussie et mises en relation avec la formation.

The purpose of the study is to describe the perceptions of freshly graduated teachers in physical and health education regarding their professional competencies. At the end of their initial training, 16 graduates in physical and health education were questioned using semi-structured interviews. The results indicate that competencies relating to designing and monitoring teaching activities are the most significant competencies at the beginning of their career, whereas the one related to use of technology is considered less important. Also questioned on their level of mastery, they consider being most comfortable in communicating clearly with the students and acting ethically. However, assessing students and adapting instruction for students with special needs seem to be less well mastered. Some convergent and divergent perceptions are discussed in light of successful professional integration and teacher education.

\section{INDEX}

Mots-clés : compétences professionnelles, éducation physique et à la santé, finissants, formation initiale d'enseignants, insertion professionnelle, perceptions des nouveaux enseignants

\section{AUTEURS}

\section{JOHANNE GRENIER}

Département de kinanthropologie

Université du Québec à Montréal

grenier.johanne@uqam.ca

\section{MARIE-CLAUDE RIVARD}

Département des sciences de l'activité physique

Université du Québec à Trois-Rivières

Marie-claude.rivard@uqtr.ca 


\section{CHARLOTTE BEAUDOIN}

École des sciences de l'activité physique

Université d'Ottawa

cbeaudoi@uottawa.ca

\section{SYLVAIN TURCOTTE}

Faculté d'éducation physique et sportive Université de Sherbrooke

Sylvain.turcotte@USherbrooke.ca

\section{MYLĖNE LEROUX}

Département des sciences de l'éducation Université du Québec en Outaouais

mylene.leroux@uqo.ca 